\title{
Massive Bilateral Serous Retinal Detachment in a Case of Hypertensive Chorioretinopathy
}

\author{
Luis Villalba-Pinto ${ }^{a} \quad$ M. Ángeles Hernández-Ortega ${ }^{a}$ \\ F. Javier Lavid de los Mozos ${ }^{a}$ Isabel Pascual-Camps ${ }^{b}$ \\ Rosa Dolz-Marco ${ }^{b}$ J. Fernando Arevaloc, ${ }^{c}$ Roberto Gallego-Pinazo ${ }^{b}$ \\ ${ }^{a}$ Department of Ophthalmology, Hospital Punta Europa, Algeciras, and ${ }^{b}$ Unit of Macula, \\ Department of Ophthalmology, University and Polytechnic Hospital La Fe, Valencia, Spain; \\ ${ }^{c}$ Retina Division, Wilmer Eye Institute, Johns Hopkins University School of Medicine, \\ Baltimore, Md., USA; ${ }^{d}$ King Khaled Eye Specialist Hospital, Riyadh, \\ Kingdom of Saudi Arabia
}

\section{Key Words}

Hypertensive chorioretinopathy · Serous retinal detachment · Optical coherence tomography

\begin{abstract}
Introduction: Systemic high blood pressure is related to a variety of retinal manifestations. We present an atypical case of hypertensive chorioretinopathy with massive bilateral serous retinal detachment. Case Report: A 26-year-old male with a genitourinary malformation and secondary grade IV chronic kidney failure as well as high blood pressure complained of acute vision loss. Dilated fundus examination evidenced a bilateral serous retinal detachment with macular involvement. The patient was unresponsive to oral antihypertensive therapy and dialysis treatment. The serous retinal detachment progressively decreased after the restoration of dialysis and antihypertensive therapy. The final visual acuity was 0.50 in both eyes. Discussion: In cases of serous macular detachment, it is mandatory to rule out different systemic and ocular diseases. The presence of uncontrolled high blood pressure may produce aggressive bilateral retinal changes, thus hypertension must be under early and strict control in order to improve the visual outcomes.

(c) 2014 S. Karger AG, Basel
\end{abstract}


Villalba-Pinto et al.: Massive Bilateral Serous Retinal Detachment in a Case of Hypertensive Chorioretinopathy

\section{Introduction}

Systemic high blood pressure is one of the most common diseases in the industrialized countries, with an increasing prevalence due to the longer life expectancy of the population. Hypertension may cause or accelerate changes in the vascular wall of target organs such as the kidney, brain, or heart. Particularly in the eye, arterial hypertension may induce changes in the retina, the choroid, and the optic nerve. It may also cause a wide range of injuries: from mild to severe vascular narrowing to loss of visual acuity due to ischemic optic neuropathy [1].

Vascular narrowing is the primary response of retinal arteries to hypertension. Typical signs in the fundus due to hypertensive retinopathy include diffuse or focal vasoconstriction, increased vascular permeability and arteriolosclerosis with thickening of the vessel wall. These 3 events are responsible for the development of different lesions that will characterize the stages of the retinal disease: arteriovenous crossings, hard and cotton ball exudates, thrombosis, embolisms, retinal hemorrhages, papilledema, and ischemic optic neuropathy (in severe cases). In addition, the presence of macular serous retinal detachment has been reported to be a marker of malignant hypertension [2].

\section{Case Report}

A 26-year-old man was referred to our emergency department; he complained about bilateral red eye associated with a loss of visual acuity (VA) starting 1 week ago. His medical history was remarkable for grade IV chronic kidney disease secondary to genitourinary malformation and under treatment with hemodialysis. He developed secondary hypertension, which was managed with oral antihypertensive drugs.

His VA was 'hand motions' in both eyes. The anterior segment examination revealed bilateral subconjunctival hemorrhage with no signs of intraocular inflammation. Dilated fundus examination evidenced serous retinal detachment with macular involvement (fig. 1ad). No retinal tears were observed in the peripheral retina examination with scleral depression. However, curvilinear whitish lesions scattered throughout the peripheral retina were observed. Optical coherence tomography (OCT) scans confirmed the presence of subretinal fluid in the macular region (fig. 1e-f).

The patient was uncompliant to oral antihypertensive therapy and dialysis treatment. His blood pressure measured 180/110 mm Hg. A systemic workup was conducted with the only relevant finding of a significantly increased creatinine value $(17 \mathrm{mg} / \mathrm{dl})$. Oral antihypertensive therapy and dialysis were initiated in order to control the kidney failure and the arterial hypertension.

One week later, his VA improved to 0.3 in both eyes. Dilated fundus examination demonstrated a significant regression of the retinal detachment. After 2 weeks, his VA improved to 0.5 in both eyes, showing a subcomplete resolution of the exudative detachment in his right eye and a persistence of subretinal fluid in the macula and the inferior retina in his left eye (fig. 2a-f).

As the retina reattached, splinter hemorrhages and retinal vascular tortuosity were observed, thus supporting the hypertensive origin of the retinopathy. Likewise, the whitish areas remained unchanged (fig. 2c, d), especially in the retinal periphery. We assume that this might be secondary to ischemia of the underlying retinal pigment epithelium and the choroid. A longer follow-up could not be performed because the patient did not show up for his scheduled visits. 
Villalba-Pinto et al.: Massive Bilateral Serous Retinal Detachment in a Case of Hypertensive Chorioretinopathy

\section{Discussion}

The potential causes of serous retinal detachment include a variety of conditions that can be classified into idiopathic, congenital, postsurgical, inflammatory, uveitic, hematologic, vascular, and neoplastic [3]. A complete examination, anamnesis and workup should be undertaken in patients with serous retinal detachment.

The main differential diagnostic possibility in the case we reported was the VogtKoyanagi-Harada syndrome, a systemic inflammatory disease with ocular, neurological and skin involvement, caused by an immune reaction to antigens of melanic cells [4]. It usually affects young women. Its hallmark is the presence of lobulated bilateral serous retinal detachment involving the macula, and associated with variable degrees of vitreous cells and flare. The absence of extraocular manifestations, altogether with the absence of increased vitreal cellularity, made us rule out this possibility.

Hypertension can cause ischemic changes due to a disruption of the external bloodretinal barrier. These changes can be explained by the increase of endogenous vasoconstrictor agents, leading to vasoconstriction and fibrinoid necrosis of the choroidal vessels. This leads to ischemia of the overlying retinal pigment epithelium (Elschnig spots). Ischemia of the pigment epithelium allows leakage into the subretinal space (serous retinal detachment) $[1,5]$.

In our case, the serous retinal detachment resolved following a normalization of blood pressure. Fundus findings were suggestive of hypertensive retinopathy such as splinter hemorrhages, vascular sclerosis and tortuosity. The peripheral white spots found in the fundus examination may correspond to ischemic areas of the RPE or Elschnig spots (fig. 2c, d).

Cases of hypertensive chorioretinopathy with serous retinal detachment have been previously reported and are related to malignant hypertension [2], preeclampsia [6], eclampsia [7], hemolysis, elevated liver enzymes, low platelet count (HELLP) syndrome during pregnancy [8], primary pulmonary hypertension [9], and in Castleman's disease [10]. In these cases, the subretinal exudation was typically limited to the macular area without any massive serous bilateral detachment.

In summary, we report the case of a young man with massive bilateral retinal detachment secondary to hypertensive chorioretinopathy due to an acute decompensation of his chronic renal failure after treatment discontinuation. It is mandatory for the ophthalmologist to recognize the ocular manifestations potentially relating to life-threatening systemic diseases.

\section{Acknowledgements}

We would like to thank Catalina Barrera Campanario and Ma Ángeles Gallardo Blanco for their valuable assistance in the acquisition of images for this paper.

\section{References}

1 Sánchez Salorio M, Pazos Gonzalez B: Enfermedades cardiovasculares; in Sánchez Salorio M, Díaz-Llopis M, Benítez del Castillo JM, Rodríguez Ares MT (eds): Manifestaciones oftalmológicas de las enfermedades generales. Madrid, Sociedad Española de Oftalmología, 2001, pp 19-46.

-2 Shukla D, Ramchandani B, Vignesh TP, Rajendran A, Neelakantan N: Localized serous retinal detachment of macula as a marker of malignant hypertension. Ophthalmic Surg Lasers Imaging 2010;9:1-7. 


\begin{tabular}{l|l}
\hline Case Rep Ophthalmol 2014;5:190-194 \\
\hline DOI: 10.1159/000364942 & $\begin{array}{l}\text { ○ 2014 S. Karger AG, Basel } \\
\text { www.karger.com/cop }\end{array}$ \\
\hline
\end{tabular}

Villalba-Pinto et al.: Massive Bilateral Serous Retinal Detachment in a Case of Hypertensive Chorioretinopathy

3 Anand R, Tasman WS: Nonrhegmatogenous retinal detachment; in Ryan SJ (ed): Retina, ed 2, St. Louis, Mosby, 1994, pp 2463-2488.

-4 Read RW, Holland GN, Rao NA, Tabbara KF, Ohno S, Arellanes-Garcia L, Pivetti-Pezzi P, Tessler HH, Usui M: Revised diagnostic criteria for Vogt-Koyanagi-Harada disease: report of an international committee on nomenclature. Am J Ophthalmol 2001;131:647-652.

5 Kishi S, Tso MO, Hayreh SS: Fundus lesions in malignant hypertension. A pathologic study of experimental hypertensive choroidopathy. Arch Ophthalmol 1985;103:1189-1197.

6 Day H, Burns J, Bosio P: A case of bilateral serous retinal detachments in severe pre-eclampsia. J Obstet Gynaecol 2008;28:534-535.

-7 Varghese S, Koshy J, Avasthi K: Bilateral serous retinal detachment in a case of eclampsia. Health 2012;4:1314.

8 Tranos PG, Wickremasinghe SS, Hundal KS, Foster PJ, Jagger J: Bilateral serous retinal detachment as a complication of HELLP syndrome. Eye 2002;16:491-492.

-9 Sánchez-Sevila JL, Martínez-Rubio M, López-Meca I, Belmonte-Martínez J: Exudative retinal detachment and primary pulmonary hypertension (in English, Spanish). Arch Soc Esp Oftalmol 2013;88:189-192.

$\checkmark 10$ Liu DTL, Shields JA, Li CL, Ng KH, Lam DSC: Hypertensive choroidopathy in Castleman's disease. Graefes Arch Clin Exp Ophthalmol 2011;249:1901-1903.
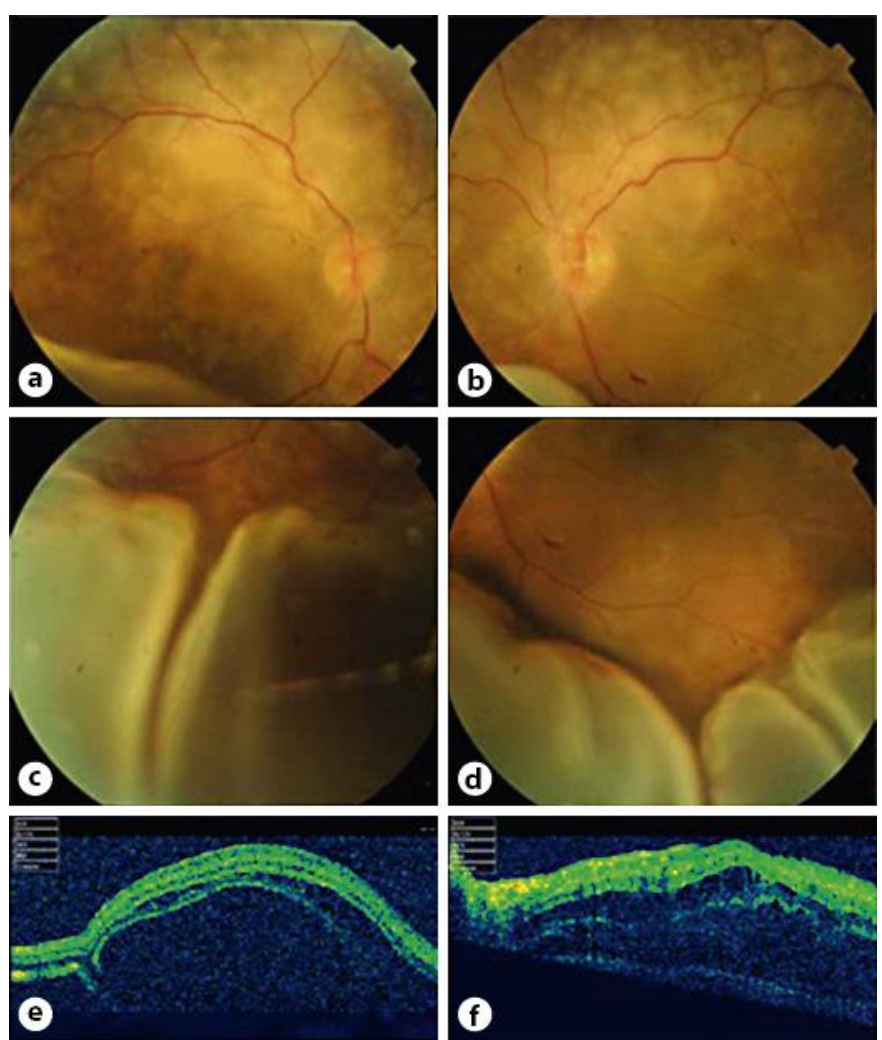

Fig. 1. Complete macula-off serous retinal detachment with lower lobulated giant exudative retinal elevation (a-d). OCT scans showing serous macular detachment in both eyes $(\mathbf{e}, \mathbf{f})$. 
Case Reports in

Ophthalmology

\begin{tabular}{l|l}
\hline Case Rep Ophthalmol 2014;5:190-194 \\
\hline DOI: 10.1159/000364942 & $\begin{array}{l}\text { ( 2014 S. Karger AG, Basel } \\
\text { www.karger.com/cop }\end{array}$ \\
\hline
\end{tabular}

Villalba-Pinto et al.: Massive Bilateral Serous Retinal Detachment in a Case of Hypertensive Chorioretinopathy
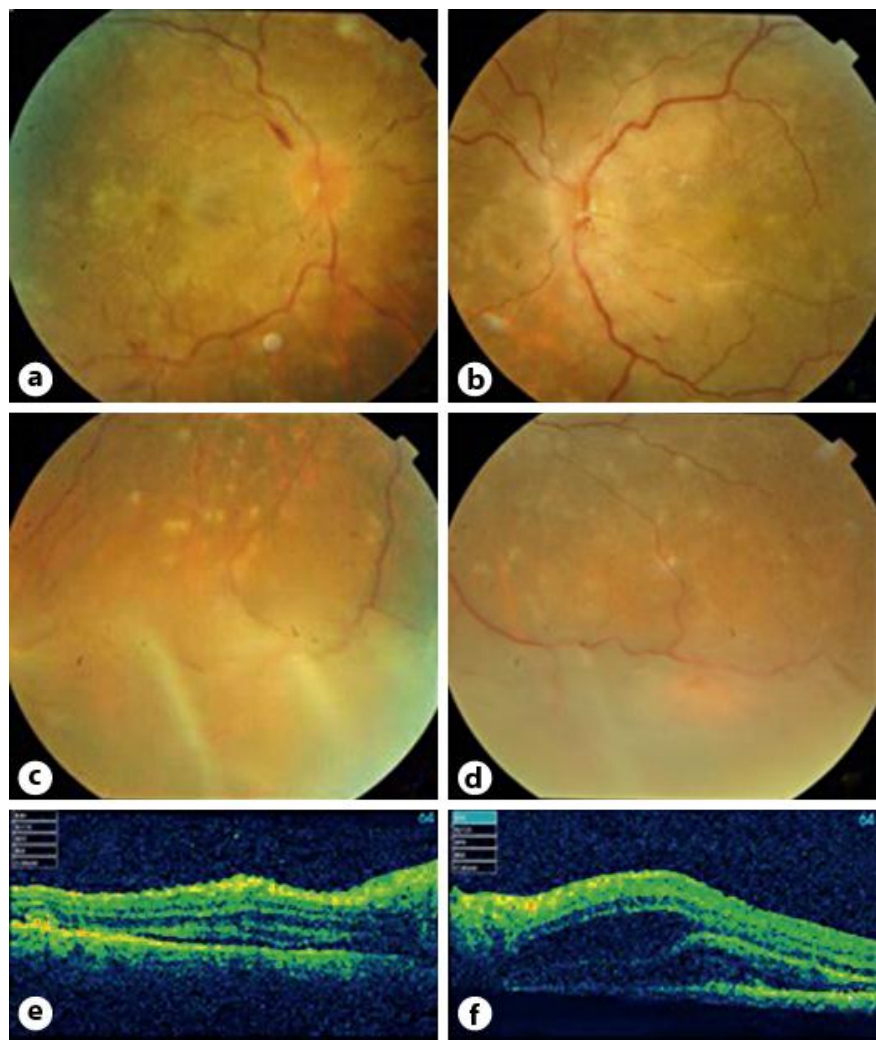

Fig. 2. Partial resolution of the bullous retinal detachment. Elschnig peripheral spots and other signs of hypertensive retinopathy are observed (a-d). OCT scans showing a subcomplete regression of macular serous detachment in both eyes $(\mathbf{e}, \mathbf{f})$. 